\title{
Criminologie
}

\section{De l'évaluation clinique au calcul de probabilité : le recours aux outils actuariels dans les pénitenciers canadiens}

\section{Bastien Quirion et Lisa D’Addese}

Volume 44, numéro 2, automne 2011

URI : https://id.erudit.org/iderudit/1005798ar

DOI : https://doi.org/10.7202/1005798ar

Aller au sommaire du numéro

Éditeur(s)

Les Presses de l’Université de Montréal

ISSN

0316-0041 (imprimé)

1492-1367 (numérique)

Découvrir la revue

Citer cet article

Quirion, B. \& D’Addese, L. (2011). De l'évaluation clinique au calcul de probabilité : le recours aux outils actuariels dans les pénitenciers canadiens.

Criminologie, 44(2), 225-250. https://doi.org/10.7202/1005798ar
Résumé de l'article

Dans le cadre du présent article, nous abordons la question du virage actuariel qui s'est manifesté dans le champ de la pénalité, particulièrement en ce qui concerne la multiplication des outils d'évaluation actuarielle qui sont désormais mis à la disposition des intervenants correctionnels. Les objectifs de cet article sont au nombre de trois. Tout d'abord, nous décrivons l'ampleur et la nature des transformations qui se sont opérées au cours des deux dernières décennies en ce qui a trait aux outils utilisés par les intervenants correctionnels canadiens. Par la suite, nous délimitons sur le plan conceptuel et théorique les principaux enjeux de ces transformations techniques, particulièrement en ce qui concerne la réduction du pouvoir discrétionnaire accordé aux intervenants. Finalement, nous analysons le discours des intervenants afin de mieux évaluer comment ces modifications ont été reçues par les intervenants eux-mêmes dans le cadre de leur pratique professionnelle. Il s'agit en fait d'évaluer l'impact de l'introduction et de l'utilisation d'une nouvelle gamme d'outils actuariels sur les pratiques correctionnelles. 


\title{
De l'évaluation clinique au calcul de probabilité: le recours aux outils actuariels dans les pénitenciers canadiens
}

\author{
Bastien Quirion \\ Département de criminologie \\ Université d'Ottawa \\ bquirion@uottawa.ca \\ Lisa D'Addese \\ Université de Toronto \\ lisa.daddese@utoronto.ca
}

\begin{abstract}
RÉSUMÉ - Dans le cadre du présent article, nous abordons la question du virage actuariel qui s'est manifesté dans le champ de la pénalité, particulièrement en ce qui concerne la multiplication des outils d'évaluation actuarielle qui sont désormais mis à la disposition des intervenants correctionnels. Les objectifs de cet article sont au nombre de trois. Tout d'abord, nous décrivons l'ampleur et la nature des transformations qui se sont opérées au cours des deux dernières décennies en ce qui a trait aux outils utilisés par les intervenants correctionnels canadiens. Par la suite, nous délimitons sur le plan conceptuel et théorique les principaux enjeux de ces transformations techniques, particulièrement en ce qui concerne la réduction du pouvoir discrétionnaire accordé aux intervenants. Finalement, nous analysons le discours des intervenants afin de mieux évaluer comment ces modifications ont été reçues par les intervenants eux-mêmes dans le cadre de leur pratique professionnelle. Il s'agit en fait d'évaluer l'impact de l'introduction et de l'utilisation d'une nouvelle gamme d'outils actuariels sur les pratiques correctionnelles.
\end{abstract}

MOTS-CLÉs - Intervention correctionnelle, évaluation de risque, outils actuariels, pouvoir discrétionnaire, Canada.

\section{Introduction}

Le champ de la pénalité est un champ en constante évolution qui fluctue au gré des transformations sociales et politiques plus générales. 
Dans le cadre de cet article, nous proposons d'explorer les transformations qui se sont manifestées dans un secteur d'activités particulier du champ pénal qui est celui de l'intervention correctionnelle. On regroupe dans ce secteur d'activités l'ensemble des interventions cliniques offertes dans le contexte de la prise en charge correctionnelle et qui ont pour finalité de transformer les détenus de façon à réduire leur propension aux comportements criminels. L'intervention correctionnelle regroupe à la fois les activités tournant autour de l'évaluation clinique du détenu, ainsi que les activités strictement thérapeutiques dont l'objectif est de modifier les conduites, les traits de personnalité ou les attitudes du détenu.

À l'instar du champ pénal plus général, ce secteur d'activités a aussi été touché par des transformations majeures au cours des dernières décennies, conduisant certains analystes à associer ces mutations à l'émergence d'une nouvelle pénologie (Feeley et Simon, 1992; 1994). Ces transformations se sont manifestées, en ce qui concerne le système correctionnel canadien, par une multiplication des programmes d'inspiration cognitiviste, par une responsabilisation accrue des détenus et par l'introduction des outils d'évaluation actuariels (Quirion, 2006; 2009). Dans cet article, nous limiterons notre propos aux transformations concernant les outils mis à la disposition des intervenants correctionnels canadiens pour évaluer les détenus qui leur sont confiés. Plus précisément, nous proposons d'analyser l'impact de l'introduction des outils actuariels sur les pratiques professionnelles des intervenants qui travaillent au Service correctionnel du Canada.

Les objectifs de cet article sont au nombre de trois. Tout d'abord, nous décrivons l'ampleur et la nature des transformations qui se sont opérées au cours des deux dernières décennies en ce qui concerne les outils d'évaluation utilisés par les intervenants correctionnels canadiens. Après avoir souligné l'importance que revêt la pratique prédictive au sein des agences correctionnelles, nous décrivons brièvement le virage actuariel qui s'est opéré dans le système correctionnel canadien. Dans un deuxième temps, nous proposons d'établir une distinction plus précise entre les principales catégories d'outils d'évaluation qui sont utilisés en milieu correctionnel. Ces considérations conceptuelles nous permettent alors de mieux souligner l'un des principaux enjeux de ce virage actuariel, en l'occurrence celui de la réduction du pouvoir discrétionnaire accordé aux intervenants correctionnels. Et finalement, à la lumière de ces considérations conceptuelles et théoriques, nous 
analysons le discours des intervenants afin d'évaluer l'impact de l'introduction des outils actuariels sur leur pratique professionnelle. Considérant que l'un des principaux enjeux du virage actuariel semble être la réduction du pouvoir discrétionnaire des intervenants, notre analyse est menée en s'inspirant de l'hypothèse selon laquelle on devrait rencontrer une certaine résistance des intervenants en ce qui concerne l'introduction des outils actuariels.

\title{
Système de prédiction et gestion de l'information
}

\author{
While the prediction of violent behavior shares many \\ features with the prediction of other forms of human conduct, \\ the potential consequences of its misapplication \\ give it a priority in professional and ethical concern.
} (Monahan, 1981: 36)

Dans une perspective organisationnelle, on peut considérer la prise en charge correctionnelle comme un parcours institutionnel ponctué d'une série de décisions prises par les intervenants à l'égard des détenus qui se retrouvent sous leur autorité (Gottfredson et Gottfredson, 1988). Les intervenants qui évoluent dans le système correctionnel canadien sont ainsi appelés à prendre des décisions par rapport à deux aspects complémentaires de l'activité correctionnelle, soit l'aspect sécuritaire et l'aspect réhabilitatif. Sur le plan de la sécurité, le système correctionnel est par définition appelé à gérer des individus qui sont reconnus comme représentant une menace ou un préjudice éventuel pour la communauté 1. Que ce soit dans une perspective plus traditionnelle d'évaluation de la dangerosité, ou une perspective plus contemporaine de gestion des risques, la question de la régulation des individus et des conduites pouvant représenter une menace pour la société demeure la principale tâche des intervenants correctionnels. Ces intervenants seront en effet appelés dans le cadre de leurs activités professionnelles à prendre des décisions qui tiennent compte de la sécurité du public: détention préventive, autorisation de sortie temporaire, mise en liberté provisoire, octroi de libération conditionnelle. En ce qui concerne la réhabilitation, les intervenants seront ici appelés à prendre des décisions quant au

1. La mission du Service correctionnel du Canada fait explicitement référence à cet objectif de protection du public: "Contribuer à la protection de la société en incitant activement et aidant les délinquants à devenir des citoyens respectueux des lois» (www. csc-scc.gc.ca). 
potentiel de réinsertion sociale que représentent les justiciables qui se retrouvent sous leur supervision. Ces décisions portent alors sur l'évaluation des besoins du bénéficiaire, l'élaboration d'un agenda clinique et les autorisations de retour en communauté.

Ces décisions seront prises à la lumière d'un certain nombre d'informations et de renseignements auxquels auront accès les intervenants correctionnels. Ces informations se retrouvent déjà dans le dossier du détenu, ou bien seront compilées directement par les intervenants qui seront appelés à travailler auprès de la clientèle. Sur le plan conceptuel, ces décisions seront prises dans le cadre de ce qu'on peut définir comme un système de prédiction, soit un système de production et d'organisation des informations qui seront mises à la disposition des intervenants afin de les aider à mieux évaluer l'impact de leurs propres décisions (Briggs et Wirt, 1965 : 170). Traditionnellement, le système de prédiction reposait sur une finalité double, soit une finalité explicative et une finalité strictement prédictive. Dans un premier temps, cette organisation de l'information visait à mieux expliquer, sur une base empirique et théorique, certains comportements humains. C'est dans cet esprit que, pendant longtemps, l'évaluation du potentiel de dangerosité devait passer au préalable par une compréhension empirique et théorique de la conduite dangereuse. L'expert était en mesure de prédire les comportements à venir d'un individu, puisqu'il était en mesure d'identifier les facteurs ou les causes qui pouvaient conduire à une plus grande propension aux comportements violents. Dans un deuxième temps, l'intervenant pouvait ensuite utiliser ces informations afin de prédire les agissements futurs d'un individu en particulier. Il s'agit alors de combiner les informations obtenues afin de se prononcer, en suivant une finalité strictement prédictive, sur les conséquences des décisions prises par les intervenants correctionnels. Mises en commun, ces deux finalités permettaient alors aux intervenants de mieux encadrer les détenus par une gestion organisée des informations produites sur chacun des individus pris en charge par les agences correctionnelles.

\section{Le virage actuariel}

Bien que la tâche de l'intervenant correctionnel ait peu changé quant à la nature et à la portée des décisions qu'il est appelé à prendre, on enregistre depuis quelques décennies des transformations majeures en ce qui concerne les outils mis à sa disposition dans le cadre de son 
travail, en particulier les outils qu'on utilise pour produire des informations sur les détenus. Ces transformations se seraient manifestées particulièrement à travers la multiplication des grilles d'évaluation statistiques ou actuarielles qui vont remplacer graduellement les techniques plus traditionnelles d'évaluation des cas (Glaser, 1985). L'engouement pour cette nouvelle gamme d'outils actuariels semble alors moussé, dès le début des années 1980, par le besoin manifeste de lutter contre l'arbitraire et le caractère discrétionnaire des décisions prises au sein du système pénal (Walker, 1993). Ces transformations coïncident donc avec le souci de réduire la marge de manœuvre accordée traditionnellement aux intervenants correctionnels.

Cet engouement pour l'utilisation des grilles statistiques n'est certainement pas étranger non plus au prosélytisme dont a fait preuve un groupe de chercheurs convaincus de la supériorité des outils actuariels par rapport au jugement clinique traditionnel (Meehl, 1954; Monahan, 1981; Groove et Meehl, 1996; Grove et al., 2000). Les travaux de recherche menés dans ce domaine ont dès lors permis de mettre au point toute une panoplie de grilles actuarielles qui seront implantées dans les agences correctionnelles canadiennes à compter du milieu des années 1980 (Beech et al., 2003). Dénonçant le caractère soi-disant intuitif du jugement clinique de l'intervenant, certains chercheurs vont décrire le virage actuariel comme le passage d'une première génération d'outils d'évaluation, qui reposeraient essentiellement sur le jugement subjectif de l'intervenant, à une seconde génération d'outils de nature statistique, dont le caractère davantage objectif est dès lors perçu comme un gage de précision, d'uniformisation et d'efficacité (Bonta, 1996). Cet enthousiasme pour le recours aux grilles actuarielles n'est cependant pas partagé par tous. En réaction à cet emballement pour l'utilisation de grilles statistiques, certains chercheurs vont plutôt soulever des doutes quant à la prétendue supériorité actuarielle, tant sur le plan de l'efficacité prédictive des outils que des fondements méthodologiques de l'approche (Hart et al., 2007; Litwack, 2001)2.

Dans une perspective plus générale, on peut aussi associer cette montée de l'actuarialisme à l'importance prise par le langage du risque dans la façon de penser désormais l'intervention auprès des populations

2. Pour une présentation plus en profondeur du débat concernant l'efficacité prédictive des grilles actuarielles et du jugement clinique, voir Litwack et Schlesinger (1999) ainsi que Harris (2006). 
marginales (Cartuyvels, 2008; Kemshall 2003). La montée de la société du risque aura en effet pour conséquence de redéfinir les problèmes sociaux traditionnels selon de nouveaux critères, délaissant le déterminisme social au profit d'une logique actuarielle. Cette tendance serait à ce point lourde que certains y verraient les signes d'une remise en question du modèle déterministe hérité du $\mathrm{XIX}^{\mathrm{e}}$ siècle, au profit d'un modèle probabiliste de saisie du monde (Hacking, 1990). C'est ainsi que dans des champs d'intervention aussi variés que celui de la santé mentale, de la toxicomanie ou de la pauvreté, le nouveau langage du risque aura pour effet de reformuler les problèmes sociaux selon une logique statistique, tout en redéfinissant le rôle des intervenants et en limitant de façon significative leur autonomie professionnelle (Fitzgibbon, 2007; HorlickJones, 2005). Cette actuarialisation du champ de l'intervention se traduit dès lors par des nouveaux impératifs d'efficacité et d'uniformisation des pratiques, par lesquels on demande désormais aux intervenants d'utiliser des outils d'évaluation plus rigoureux et standardisés, afin de mieux mesurer et de mieux contrôler les risques socio-sanitaires, tels que le risque de récidive ou de comportement violent.

Selon Nikolas Rose (2002), le recours à ces outils actuariels standardisés pourrait aussi représenter un avantage pour les autorités correctionnelles du fait qu'ils assurent un meilleur contrôle non seulement sur les délinquants qui y sont soumis, mais aussi sur les intervenants qui sont appelés à les appliquer. À ce titre, les outils actuariels pourraient aussi représenter un avantage du fait qu'ils permettraient une meilleure régulation de l'activité professionnelle au sein du système correctionnel. En assurant une plus grande standardisation des pratiques, ces outils permettraient d'atteindre une finalité double, soit celle d'assurer une meilleure gestion des populations carcérales, et celle plus subtile de gérer plus efficacement le travail des intervenants eux-mêmes. À cet égard, l'efficacité de ces nouveaux instruments ne se mesurerait plus seulement par rapport à la précision des résultats obtenus, mais aussi par rapport à la standardisation des pratiques évaluatives et prédictives. On ne chercherait plus seulement à mieux prédire la récidive, mais aussi à mieux prédire le déroulement des pratiques d'évaluation elles-mêmes.

On peut donc évoquer, comme point de départ de cet article, la présence d'une révolution technique - soit le virage actuariel - à défaut de pouvoir pour l'instant explorer l'hypothèse d'une reconfiguration plus en profondeur des fonctions traditionnellement attribuées aux agences correctionnelles. Lobjet de cette contribution est donc de 
décrire la nature de ces transformations du point de vue technique, pour ensuite évaluer l'impact de l'introduction de ces nouveaux outils sur la pratique des intervenants correctionnels, particulièrement en ce qui concerne leur autonomie professionnelle. Mais avant d'évaluer cet impact, il semble important de mieux distinguer sur le plan conceptuel les différentes catégories d'outils utilisés au sein du système correctionnel canadien et de cerner les principaux enjeux cliniques liés à l'introduction des outils actuariels.

\section{Évaluation clinique vs évaluation actuarielle}

It was thus the subsequent convergence of the collapse of faith in clinical diagnosis of the one hand, and the introduction of this sophisticated computer technology on the other that began to make actuarial prediction a more viable penal strategy. (Pratt, 1997: 175)

Afin de bien évaluer l'impact de ce virage actuariel, il est nécessaire au départ de bien distinguer ces deux catégories d'outils que l'on désigne en général comme les outils cliniques traditionnels et les outils actuariels. Cet exercice nous apparaît d'autant plus nécessaire que les efforts de catégorisation que l'on retrouve dans la littérature scientifique souffrent en général d'un manque de rigueur sur le plan conceptuel. On retrouve de nombreux travaux portant sur l'analyse comparative de l'efficacité des grilles cliniques et actuarielles, mais en général on ne réussit pas à y établir de façon rigoureuse les critères pour distinguer ces deux catégories d'outils (Dawes et al., 1989; Grove et Meehl, 1996; Grubin, 2004; Guay, 2006; Litwack, 2001). On réduit souvent le caractère actuariel à l'utilisation d'un outil mécanique, négligeant ainsi l'aspect probabiliste qui caractérise toute mesure actuarielle. Cette absence de configuration conceptuelle rigoureuse induit ainsi une certaine confusion lorsque vient le temps de classer certains outils particuliers dans l'une ou l'autre de ces catégories.

En empruntant librement à Monahan (1981) sa description du processus d'évaluation de la dangerosité $e^{3}$ et en s'inspirant aussi du concept

3. Monahan (1981: 42) formule les différentes étapes à partir des trois affirmations que comporte tout exercice de prédiction. Ces affirmations concernent le fait que l'individu évalué cumule certains traits ou caractéristiques; que ces caractéristiques sont associées à la propension ou à la probabilité d'une récidive violente; et que le niveau de risque ou de dangerosité est suffisamment important pour justifier une intervention préventive. 
de système de prédiction tel qu'exposé plus haut, on peut distinguer trois étapes qui, une fois combinées, forment cette tâche qui consiste à prédire les comportements humains: 1) la production et la sélection des informations; 2) l'organisation et la combinaison des informations; et 3) la prise de décision.

La première étape, soit la production et la sélection des informations, consiste à établir la liste des informations qui seront retenues par le système de prédiction afin d'expliquer et de prédire les comportements futurs. Il s'agit en fait de l'étape qui consiste à identifier les prédicteurs qui seront utilisés ultérieurement pour évaluer la dangerosité ou la probabilité de récidive de la clientèle correctionnelle.

La deuxième étape, soit l'organisation et la combinaison des informations, consiste à établir si les informations ainsi retenues sont associées à une plus grande propension en regard des conduites que l'on cherche à prédire. Il s'agit en fait d'organiser les informations produites ou sélectionnées lors de la première étape, de façon à créer un savoir qui soit utile dans le cadre du processus décisionnel des intervenants correctionnels. On combine selon certains critères bien précis les informations, afin de créer une certaine mise en ordre du savoir qui puisse mieux se prêter aux besoins institutionnels des agences correctionnelles. C'est l'étape au cours de laquelle l'intervenant sera appelé à mesurer de façon précise la probabilité de récidive ou à estimer le niveau de dangerosité d'un détenu.

Et finalement, la prise de décision, qui constitue la troisième étape du processus de prédiction des conduites. Cette étape consiste essentiellement à décider, en s'appuyant sur les résultats obtenus lors de la combinaison des informations, si le niveau de dangerosité ou la probabilité de récidive justifie la mise en place de mesures spécifiques.

Alors que les deux premières étapes renvoient à des tâches techniques qui peuvent être réalisées, en principe, dans un contexte plus neutre en ce qui concerne la mission politique de l'institution correctionnelle, la troisième étape implique essentiellement une prise de décision de nature politique. Cette décision finale repose en fait sur le choix d'un seuil (niveau de risque ou de dangerosité) à partir duquel l'institution jugera qu'il est justifié de mettre en place des mesures préventives ou de refuser un retour en communauté. Les outils qui seront mis à la disposition des intervenants pour prédire les comportements à venir vont donc se distinguer, sur le plan technique, à partir des deux activités spécifiques que sont la sélection des informations et leur combinaison. Ainsi, la 
distinction entre les techniques d'évaluation clinique traditionnelle de la première génération et les techniques actuarielles de la seconde génération repose en fait sur la façon dont ces deux premières activités seront menées. La distinction conceptuelle proposée repose donc sur la façon dont les étapes de la production et de l'organisation des informations nécessaires à la pratique prédictive seront menées. On peut ainsi, en s'appuyant sur la description de ces deux premières étapes de la prédiction des comportements, établir sur le plan conceptuel les principaux critères permettant de distinguer trois catégories d'outils d'évaluation.

\section{Évaluation clinique traditionnelle}

L'évaluation clinique dite traditionnelle renvoie à toute forme de prédiction qui confère à l'intervenant une importante marge de manœuvre dans la façon de conduire l'évaluation. Il existe toutefois différents degrés de liberté dans la façon dont l'intervenant mènera l'évaluation, passant de l'évaluation essentiellement basée sur l'expérience clinique de l'intervenant à des formes plus structurées d'évaluation qui s'appuient sur des résultats empiriques validés par la recherche ${ }^{4}$.

En ce qui concerne la première étape, la production et la sélection des informations va varier d'une évaluation à l'autre, puisque l'évaluateur jugera pour chacun des cas de la pertinence des informations retenues. Il n'existe pas de listes préétablies des prédicteurs qui seront retenus, puisque c'est l'intervenant qui décide, sur une base individuelle et propre à chaque cas, des traits ou caractéristiques qui seront pris en considération dans l'exercice prédictif. La formule classique consiste à prendre en considération les informations disponibles concernant l'individu sous observation, pour éventuellement sélectionner les facteurs qui apparaissent pertinents aux yeux de l'évaluateur pour mener à terme sa démarche prédictive. Contrairement à ce que prétendent les promoteurs de l'évaluation actuarielle (Monahan, 1981; 96), on ne peut ramener la question de l'évaluation clinique à la simple expression d'un jugement intuitif. L'évaluation clinique s'appuie en fait sur l'expertise théorique et empirique de l'intervenant appelé à conduire l'évaluation,

4. On regroupe sous le terme de anamnestic risk assessment l'ensemble des méthodes d'évaluation de la dangerosité qui, bien qu'elles s'appuient sur le jugement clinique des intervenants, s'inspirent de résultats de recherche et sont généralement plus structurées que les évaluations cliniques traditionnelles (Andrews, Bonta et Wormith, 2006). 
pouvant ainsi témoigner d'une véritable rigueur tant sur le plan de la sélection que de la combinaison des informations ${ }^{5}$. Cette clarification nous apparaît d'autant plus nécessaire que chez les tenants de l'approche actuarielle, cette référence à l'intuition véhicule souvent une appréciation péjorative.

En ce qui concerne la deuxième étape, l'évaluateur combine les facteurs retenus en s'inspirant d'une grille théorique particulière ou de sa propre expérience clinique. Il n'existe donc pas de méthode précise de combinaison des facteurs qui sera appliquée de façon systématique pour chacun des cas à évaluer. Les liens entre les prédicteurs et le comportement à prédire sont donc de l'ordre de la prédisposition, plutôt que du registre de la probabilité statistique. À ce titre, l'évaluation clinique traditionnelle permet de se prononcer sur la propension de l'individu à passer à l'acte et implique un important pouvoir discrétionnaire, qui se manifeste à la fois dans le choix des informations retenues et dans la façon de combiner ces informations. La qualité des évaluations repose donc essentiellement sur l'expérience et les aptitudes cliniques de l'évaluateur.

\section{Évaluation actuarielle}

On regroupe généralement sous la catégorie de l'évaluation actuarielle toute évaluation qui repose principalement sur l'application d'une grille de calcul statistique de la probabilité de récidive des sujets évalués.

En ce qui concerne l'étape initiale de la production et de la sélection des informations, la grille actuarielle dicte de façon stricte et précise les informations qui devront être recueillies auprès du détenu. En principe, l'intervenant appelé à mener l'évaluation ne bénéficie que d'une étroite marge de manœuvre quant au choix des prédicteurs qui seront pris en compte dans l'exercice de la prédiction. Les particularités propres à chacun des cas ne seront donc pas prises en compte par ce type d'évaluation. En ce qui concerne la production des informations, une liste stricte des prédicteurs est établie au préalable en se basant sur les résultats d'études conduites auprès de larges échantillons de détenus,

5. À cet égard, Paul Meehl prend bien la peine, dans son ouvrage désormais classique, de nuancer quant à l'aspect intuitif du jugement clinique: «It must be stressed that "nonmechanical" is not to be identified with "intuitive" or with any mode of combining data that has the connotation of subjectivism or irrationality. It may be intuitive in special cases; on the other hand the clinician making this sort of prediction may give explicit reasons for his predictions from the data» (Meehl, 1954:16). 
pour lesquels on a établi une corrélation statistique entre certains facteurs individuels et le risque de récidive. Les informations qui seront retenues sont construites à partir d'une analyse statistique de populations qui ont préalablement traversé les étapes du processus pénal. Les prédicteurs retenus sont en fait des facteurs de risque, puisque le lien entre les informations retenues et le comportement à prédire a été validé statistiquement. La nature du lien entre les prédicteurs retenus et le comportement à prédire est essentiellement statistique, ce qui implique que ces facteurs ne peuvent être assimilés à des prédispositions qui relèveraient d'une quelconque théorie du comportement criminel.

C'est à cet égard que l'on peut évoquer le caractère a-théorique des évaluations actuarielles (Guay, 2006: 151). À ce titre, le caractère actuariel de ces outils rend caduque la fonction traditionnelle du système de prédiction qui consistait à établir les facteurs pouvant expliquer la propension de certains individus aux comportements antisociaux. Le système de prédiction, tel qu'il se déploie à l'ère de la nouvelle pénologie, se replie essentiellement sur sa fonction de prédiction des comportements futurs, en optant pour une logique statistique dans laquelle les explications théoriques s'effacent au profit du calcul de probabilité.

En ce qui concerne la seconde étape, les prédicteurs ainsi sélectionnés seront par la suite combinés selon une logique essentiellement mathématique, en ayant recours à des techniques automatisées ou mécaniques. Pour chacun des prédicteurs sélectionnés correspond un score, qui une fois combiné aux autres scores, va permettre d'établir des résultats précis concernant la probabilité de récidive du détenu ainsi évalué. Dans sa version la plus mécanique, l'outil actuariel prendra la forme d'une grille comportant une série de cases que l'intervenant sera appelé à cocher, selon que l'individu sous évaluation cumule ou non les caractéristiques énumérées. Les résultats obtenus par la combinaison de ces prédicteurs indiquent ainsi le pourcentage ou le taux de probabilité (risque) qu'un individu récidive ou commette un geste violent. Et c'est en s'appuyant sur ce niveau de risque que les intervenants seront éventuellement appelés à prendre des décisions concernant les individus évalués.

L'exemple typique de cette catégorie d'instruments est le Violence Risk Appraisal Guide (VRAG) qui a été élaboré au Canada dans les années 1990 (Harris et al., 1993; Quinsey et al., 2006). Cet outil a été conçu afin d'établir la probabilité de récidive violente des détenus remis en liberté. 


\section{Outils hybrides}

Outre ces deux catégories d'outils de prédiction généralement reconnus, on peut constituer une catégorie intermédiaire qui combine à la fois des caractéristiques de l'évaluation clinique et de l'évaluation actuarielle, mais sans nécessairement se rattacher à l'une d'elles. Il s'agit des outils hybrides qui, tout en étant assimilés à tort aux outils actuariels du fait qu'ils prennent souvent la forme d'une grille d'évaluation automatisée et rigide, conservent toutefois certaines des caractéristiques des outils d'évaluation clinique plus traditionnels, en l'occurrence en ce qui concerne la production des informations qui seront sélectionnées.

Nous regroupons ici, sous la catégorie des outils hybrides, tous les instruments psychométriques qui combinent les informations retenues selon une méthode rigoureusement mécanique, mais dont les prédicteurs retenus ne sont pas construits et sélectionnés selon une logique probabiliste. En général, ces outils combinent des facteurs de prédiction qui seront produits selon des critères cliniques et théoriques, sans qu'aucun calcul actuariel ne soit mis à contribution. Ces outils ne permettent donc pas de mesurer la probabilité de récidive, mais plutôt de brosser un portrait clinique qui éventuellement pourra être utilisé dans le processus d'évaluation et de prédiction des conduites dangereuses.

C'est le cas, entre autres, du Level of Service Inventory - Revised (LSI-R) qui a été créé dans les années 1970 et qui est utilisé par la plupart des agences correctionnelles nord-américaines (Andrews et Bonta, 1995). Cet outil regroupe des informations relevant à la fois du calcul actuariel et du jugement clinique (Maurutto et Hannah-Moffat, 2006). Bien que la combinaison des informations se déroule selon un mode mécanique et quantitatif, les résultats de l'évaluation ne peuvent être considérés comme relevant de l'ordre de la probabilité statistique.

Un autre exemple d'outil hybride est le Psychopatby Checklist - Revised (PCL-R) qui est utilisé à titre d'outil diagnostique pour établir le niveau de personnalité antisociale (Hare, 1996; Pham et Côté, 2000). Encore une fois, bien que cet outil d'évaluation se présente sous la forme d'une grille quantitative dans laquelle la liste des items doit être rigoureusement respectée, il n'en demeure pas moins un outil clinique dont la construction et la sélection des items s'appuient au départ sur une analyse empirique (mais non actuarielle) d'individus qui manifestent des troubles de la personnalité antisociale. La principale raison pour laquelle on assimile cet instrument à la logique probabiliste, c'est que 
de nombreux outils actuariels vont récupérer le score final du PCL-R pour mesurer la probabilité de récidive (Hemphill et Hare, 2004). En effet, en s'appuyant sur des analyses statistiques, certaines recherches ont démontré qu'il pouvait y avoir une corrélation statistique entre le fait d'obtenir un score élevé au PCL-R et la probabilité d'un passage à l'acte violent. En s'inspirant des résultats de ces recherches, on a donc sélectionné, au nombre des prédicteurs permettant de mesurer la probabilité de récidive, le résultat à l'évaluation du PCL-R. C'est ainsi que de nombreux outils actuariels, dont le VRAG qui a été présenté ci-haut, vont inclure dans la liste de leurs prédicteurs le score obtenu au PCL-R.

\section{Objectifs de la recherche et méthodologie}

Ces considérations conceptuelles nous autorisent dès lors à distinguer les différentes formes d'évaluation à partir du niveau de liberté qui est laissé à l'intervenant correctionnel dans la façon de mener les différentes étapes du processus d'évaluation. L'évaluation clinique traditionnelle confère à l'intervenant un important pouvoir discrétionnaire, qui se manifeste à la fois à l'étape de la sélection des informations et à l'étape de la combinaison des informations ainsi retenues. En ce qui concerne l'évaluation actuarielle, le pouvoir discrétionnaire semble au contraire réduit de façon significative, puisque la marge de manœuvre de l'évaluateur demeure très étroite lorsque vient le temps de sélectionner et de combiner les informations jugées pertinentes. La standardisation et l'automatisation des différentes étapes de l'évaluation actuarielle auraient pour conséquence de réduire de façon considérable le pouvoir discrétionnaire des intervenants, les reléguant dans certains cas à un simple rôle technique d'application mécanique des outils mis à leur disposition.

Cet amenuisement du pouvoir discrétionnaire représente pour de nombreux partisans du recours aux outils actuariels un avantage indéniable par rapport aux évaluations cliniques traditionnelles (Quinsey et al., 2006). Le partage d'une technologie et d'un langage communs permettrait, selon eux, d'uniformiser les pratiques d'évaluation, évitant ainsi le piège de la disparité des décisions à l'intérieur du système correctionnel (Andrews et al., 2006: 9).

Malgré cette méfiance généralisée par rapport à l'évaluation clinique traditionnelle, certains promoteurs des techniques actuarielles reconnaissent toutefois que le jugement clinique de l'intervenant peut com- 
porter certains avantages, pourvu qu'il soit assujetti aux résultats des calculs actuariels du niveau de risque de l'individu. C'est dans cet esprit que deux chercheurs canadiens - parmi les plus influents dans ce champ de recherche - vont reconnaître l'importance du jugement clinique, à condition qu'on en fasse un usage parcimonieux:

There is room (and a need for) professional judgment, which can serve to override these principles of assessment in the unique case. However, we must be careful that professional overrides are not used in a haphazard and irrational manner and that they do not become the preferred choice for making prediction. Rather, we should look on overrides as an opportunity to improve our [actuarial] assessment. (Andrews et Bonta, 2003: 265) ${ }^{6}$

Certains analystes évoqueront ainsi le fait que, malgré l'utilisation accrue des outils actuariels, les intervenants continuent à appuyer leurs décisions sur des informations et des croyances qui relèveraient davantage du jugement clinique traditionnel (Grove et Meehl, 1996: 299).

Bien qu'elle n'ait pas complètement relégué aux oubliettes le jugement clinique traditionnel, l'utilisation accrue des outils actuariels aurait toutefois transformé de façon importante le rôle et les tâches traditionnellement conférés aux intervenants correctionnels. C'est donc à la lumière de ces transformations que nous avons entrepris une recherche dont l'objectif était de questionner les intervenants sur l'impact de ces nouveaux outils en ce qui concerne leur pratique professionnelle. Plus précisément, nous avons invité les intervenants correctionnels à nous parler de leurs pratiques évaluatives, des outils utilisés et de leur marge de manœuvre professionnelle. Cette étude s'appuyait au départ sur l'hypothèse selon laquelle les intervenants correctionnels, confrontés au caractère jugé trop mécanique de ces nouveaux outils, auraient recours de façon informelle à l'évaluation clinique pour pondérer les résultats des outils actuariels. Il s'agit en fait d'évaluer la présence d'une certaine forme de résistance des intervenants par rapport à l'implantation et à l'utilisation de ces outils. Cette résistance prendrait alors la forme d'un ajustement clinique, c'est-à-dire d'un recours au jugement clinique pour corriger les résultats de l'évaluation actuarielle en fonction d'informations supplémentaires qui n'auraient pas été prises en

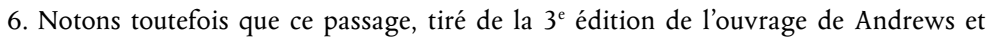
Bonta (2003), a été complètement éliminé de la version la plus récente du même ouvrage (Andrews et Bonta, 2006). On peut donc présumer que l'importance initialement accordée à l'ajustement clinique serait en voie d'être complètement évacuée. 
compte par la grille actuarielle (Litwack, 2001 : 410; Guay, 2006: 159). Cette résistance des intervenants a d'ailleurs été évoquée à plusieurs reprises par des analystes du système correctionnel canadien, que ce soit par rapport à la logique actuarielle plus générale (Vacheret et al., 1998) ou par rapport à la façon dont seront pris en compte les besoins des détenus (Hannah-Moffat, 2005). Pour reprendre la formulation de Cheliotis (2006: 327), notre intention était de vérifier à quel point les intervenants auraient fait usage de leur pouvoir discrétionnaire à titre de geste de résistance contre la montée de l'actuarialisme au sein des agences correctionnelles canadiennes.

Afin de vérifier l'hypothèse d'une éventuelle résistance des intervenants face au caractère trop rigide de l'évaluation actuarielle, nous avons mené une première série d'entrevues auprès de 12 professionnels du Service correctionnel du Canada (SCC $)^{7}$. Il s'agit en fait d'une phase préliminaire d'un projet de recherche plus important qui sera mené ultérieurement. Cette première phase nous a permis de dégager certains résultats préliminaires qui seront utilisés, lors d'entrevues subséquentes, pour mieux cerner les enjeux de la pratique évaluative en milieu correctionnel. Les participants sélectionnés ont tous été appelés, à un moment ou l'autre de leur carrière, à travailler au programme national pour délinquants sexuels et à utiliser des grilles d'évaluation actuarielles. L'échantillon retenu était composé de 4 agents de programmes correctionnels et de 8 psychologues. Les participants ont été recrutés dans différentes régions administratives du SCC, selon la répartition suivante: 6 en Ontario, 2 au Québec et 4 dans la région atlantique. Les entrevues, d'une durée variant entre une et deux heures, ont été menées de novembre 2006 à avril 2007. Nous avons alors demandé aux participants de parler de différents aspects de leur pratique professionnelle, en insistant sur deux dimensions particulières de leur pratique: l'évaluation du risque et leur perception quant au pouvoir discrétionnaire qui leur est accordé dans le cadre de cette évaluation.

Le fait que les participants à cette étude aient tous été recrutés dans le cadre du programme national pour délinquants sexuels du SCC implique toutefois des limites en ce qui concerne la généralisation des résultats à d'autres secteurs de l'intervention correctionnelle. On peut

7. Les entrevues ont été menées dans le cadre d'un projet de recherche qui a été approuvé par le Service correctionnel du Canada ainsi que par le Comité d'éthique de la recherche en sciences sociales et humanités de l'Université d'Ottawa (dossier \# 06-06-02). 
en effet présumer que pour des intervenants appelés à prendre des décisions concernant des délinquants sexuels - c'est-à-dire des délinquants pour qui la remise en liberté représente un sujet sensible aux yeux de l'opinion publique -, il peut paraître avantageux de ne pas bénéficier d'une trop grande marge de manœuvre sur le plan décisionnel. Face à la responsabilité qu'implique pour ces intervenants la possibilité de libérer un faux-négatif-soit un individu jugé à faible risque, mais qui récidive une fois libéré -, on peut comprendre que le recours à des outils plus objectifs et standardisés puisse représenter un attrait considérable. Une étude plus approfondie auprès d'une population plus étendue d'intervenants nous permettrait éventuellement de vérifier si l'attitude des intervenants quant à l'utilisation de ces grilles actuarielles est partout la même dans le système correctionnel canadien.

\section{Résultats}

Le contenu des entrevues a été analysé en tenant compte de la question initiale de recherche, à savoir l'impact du virage actuariel sur la façon dont les intervenants correctionnels évaluent leur marge de manœuvre professionnelle. Nous avons donc regroupé les extraits d'entrevue selon qu'ils traitaient de l'un des thèmes suivants: l'évaluation du risque, l'évaluation actuarielle et le pouvoir discrétionnaire accordé aux intervenants.

\section{L'évaluation du risque}

Tous les participants interrogés considèrent que l'évaluation du risque, qu'elle soit clinique ou actuarielle, représente une activité nécessaire dans le cadre de leur pratique. Bien que le temps consacré à l'évaluation du risque soit peu accaparant par rapport à d'autres activités cliniques, les participants s'accordent pour dire qu'il s'agit d'un aspect fondamental de leur pratique, puisqu'elle leur permet d'accumuler de l'information sur les détenus et les aide à prendre des décisions plus éclairées:

J'utilise l'évaluation du risque afin d'avoir une meilleure idée... un meilleur portrait de ce qui se passe... Ensuite j'élabore à partir de ces informations pour mieux comprendre où le gars en est rendu et qu'est-ce qui arrive dans sa vie... C'est un peu comme ça que je vois la chose... en quelque sorte comme de mettre en place les pièces d'un puzzle. (Participant 1 , notre traduction de l'anglais) 
À ce titre, l'évaluation du risque est considérée comme une importante activité de production et de compilation d'informations. Cette activité s'avère utile à l'intervenant dans le cadre de son travail auprès de la clientèle correctionnelle, à la fois pour sa portée explicative et pour sa portée prédictive. Par la nature objective des informations recueillies, les résultats de cette évaluation semblent rassurer les intervenants. Les participants évoquent d'ailleurs à de nombreuses reprises que l'évaluation $\mathrm{du}$ risque leur procure des informations précises et objectives sur les personnes ainsi évaluées. En général, les participants affirment avoir confiance en la fiabilité des grilles d'évaluation qui sont mises à leur disposition, en insistant sur la précision des résultats obtenus.

Certains participants, bien qu'ils soient moins nombreux, vont toutefois réprimer leur enthousiasme en évoquant le risque de stigmatisation qui est lié à ces pratiques. On souligne que les résultats obtenus lors de cette évaluation peuvent conférer aux détenus une étiquette qui aura un impact considérable sur les décisions qui seront éventuellement prises à leur égard. C'est pourquoi ces intervenants se montrent plus prudents quant à l'utilisation qu'ils feront de ces résultats d'évaluation. On mentionne que ces résultats, bien qu' ils soient utiles lors de la prise de décision, devraient toujours être considérés comme des indicateurs plutôt que comme des données brutes:

J'utilise les résultats comme des indicateurs généraux (general guidelines)... Pour être honnête avec vous, je ne leur accorde pas beaucoup de poids... Je ne crois pas que la courbe ROC soit suffisamment élevée pour qu'on puisse les considérer comme parole d'évangile... Ceci dit, je crois que ces résultats sont de bons indicateurs de ce qui peut arriver et du type de traitement que le gars a besoin. (Participant 6, notre traduction de l'anglais)

Les informations recueillies dans le cadre de ces activités d'évaluation permettent aux intervenants de mieux gérer (management) les délinquants à l'intérieur même du système correctionnel, répondant à la fois à des considérations liées à la protection du public et à la mise en place d'activités thérapeutiques. Évoquant le recours à l'évaluation du risque, ce participant indique que: «le but, je suppose, est d'évaluer le risque de récidive dans la communauté afin de mieux protéger le public... et aussi d'offrir le traitement approprié» (Participant 3, notre traduction de l'anglais).

On remarque toutefois, chez de nombreux participants, cette tendance à combiner ces deux niveaux de considération - soit celui pour 
la sécurité et celui pour le traitement -, témoignant probablement d'une confusion des intervenants quant à la dualité de leur rôle dans le processus correctionnel. On évoque ainsi, souvent dans la même phrase, des préoccupations à la fois pour la surveillance des délinquants et pour la relation d'aide:

[É]tablir les cibles du traitement [...] parce qu'il sera pertinent qu'il travaille sur tel facteur de risque qu'il devrait travailler pour diminuer le risque de récidive... pour mieux gérer le risque en communauté. (Participant 12)

On essaie de faire un usage clinique des données qui sont tirées des échelles... on essaie de faire du sens avec ce que ça veut dire pour cet individu... comment il faut intervenir... à quel moment on pense qu'il faut resserrer la surveillance... ou apporter une aide supplémentaire. (Participant 11)

Les témoignages de ces intervenants confirment ainsi l'importance qui est accordée à l'évaluation du risque au sein des institutions correctionnelles canadiennes. Bien que les autorités du Service correctionnel du Canada aient depuis longtemps adopté des politiques et des directives axées principalement sur la gestion des risques que représente le délinquant, le contenu de ces entrevues vient confirmer que cette préoccupation pour l'évaluation des risques est aussi présente dans la pratique des intervenants. À cet égard, le langage et la logique du risque semblent bien implantés dans les agences correctionnelles fédérales.

\section{Les évaluations actuarielles}

En ce qui concerne plus spécifiquement le recours aux grilles actuarielles, les intervenants interrogés considèrent pour la plupart que le virage actuariel représente un progrès significatif dans le secteur de l'intervention correctionnelle. Plusieurs participants évoquent de façon explicite la supériorité technique des outils actuariels sur les outils cliniques traditionnels, tant en ce qui concerne l'objectivité clinique que la précision des résultats obtenus:

Je crois que les outils [actuariels] sont mieux... Les gens acceptent en général qu'on doive y avoir recours... Comme les outils actuariels sont plus précis, il faut donc les utiliser. (Participant 4, notre traduction de l'anglais)

Je pense que l'actuariel, ça permet plus d'objectivité... même si ça reste encore quand même un jugement... je pense que ça rajoute une plus grande objectivité. (Participant 12) 
La supériorité technique des outils actuariels est d'autant plus acceptée que les participants soulignent à de nombreuses reprises les limites inhérentes aux évaluations cliniques traditionnelles. On considère que le risque de se tromper est plus important si on utilise une évaluation reposant davantage sur l'intuition clinique de l'intervenant. À cet égard, les intervenants interrogés semblent avoir bien intégré le discours des promoteurs des outils actuariels, en considérant que le jugement clinique implique essentiellement le recours à l'intuition:

Je crois qu'il y a un gros problème si quelqu'un n'utilise que l'évaluation clinique... ce n'est pas très précis. Nous savons que les mesures actuarielles sont beaucoup plus précises. (Participant 1, notre traduction de l'anglais)

Comme je l'ai déjà dit, l'évaluation clinique ne fonctionne pas... Pour simplifier, disons que les évaluations actuarielles fonctionnent mieux, et je crois que nous devons nous en tenir strictement aux résultats des outils actuariels. (Participant 2, notre traduction de l'anglais)

[L]a prédiction actuarielle est plus sûre que la prédiction clinique... plus structurée... avec des définitions bien établies. La prédiction clinique est plus émotive... on y va au feeling... donc, on a plus de chances de se tromper [...] Je pense qu'on a plus tendance à surestimer le risque avec une évaluation clinique. (Participant 11)

Certains intervenants vont même comparer la fiabilité des résultats obtenus lors d'une évaluation clinique à la fiabilité d'une décision aléatoire, affirmant que les évaluations traditionnelles ne vaudraient pas davantage qu'une décision prise au hasard. C'est dire à quel point les intervenants semblent ne plus avoir confiance dans les évaluations traditionnelles qui reposent principalement sur l'expertise professionnelle et clinique de l'intervenant.

Ce que la recherche a montré, c'est [qu'avec les évaluations cliniques] on était pas meilleurs que de lancer un 25 sous. (Participant 11)

[L'évaluation clinique], ce n'est pas mieux que le hasard... En fait, certaines recherches indiquent que chez les intervenants plus conservateurs, leur évaluation a donné des résultats bien pires que le hasard... pour toutes sortes de raisons. Ce qui veut dire que dans le meilleur des cas, sans le recours aux outils actuariels, la prédiction du risque de récidive équivaut à une décision prise au hasard. [Mais] avec les outils actuariels qui sont aujourd'hui disponibles, [...] la possibilité d'obtenir de meilleurs résultats que le hasard a augmenté de façon importante. (Participant 2, notre traduction de l'anglais) 
On peut donc conclure, à la lumière de ce que disent les participants, que les nouveaux outils actuariels mis en place au cours des 20 dernières années ont conquis l'assentiment général des intervenants correctionnels. La principale qualité de ces outils est qu'ils permettent de surmonter les limites qui étaient inhérentes aux outils cliniques traditionnels, tout en permettant de rassurer les intervenants quant à la précision et la fiabilité des résultats obtenus. Quelques intervenants interrogés vont toutefois souligner certains aspects de ces outils qui, de leur avis, représentent une limite ou un piège. Sans pour autant exclure le recours aux grilles actuarielles, on évoque le manque de flexibilité de ces outils: "Je crois qu'elles sont utiles pour orienter les personnes vers les bons programmes, mais je crois aussi qu'il y a le danger qu'elles soient quelquefois appliquées de façon trop rigide» (Participant 5, notre traduction de l'anglais). Mais de façon générale, tous les intervenants rencontrés se disent satisfaits des transformations techniques qui se sont manifestées au cours des dernières années en ce qui concerne les outils mis à leur disposition pour évaluer la clientèle correctionnelle. On rencontre donc très peu de résistance des intervenants face au développement et à la mise en application de ces nouvelles techniques d'évaluation du risque.

\section{Le pouvoir discrétionnaire}

Considérant que l'utilisation des grilles actuarielles réduit de façon importante la marge de manœuvre des intervenants appelés à les utiliser, nous avons interrogé les participants afin de connaître leur opinion concernant la place octroyée au pouvoir discrétionnaire dans le cadre de leur pratique. Nous les avons donc questionnés pour savoir si le recours à des grilles actuarielles leur permettait une certaine liberté professionnelle. À cet égard, les intervenants rencontrés se disent en général satisfaits de la marge de manœuvre que leur permet l'utilisation des outils actuariels. On considère que ces outils, bien qu'ils soient soumis à des standards précis en ce qui concerne leur utilisation, offrent néanmoins aux intervenants une relative indépendance quant aux décisions qui devront être prises. «Je crois que notre pouvoir discrétionnaire est très élevé» (Participant 10, notre traduction de l'anglais). Certains participants déclarent pouvoir bénéficier de toute la liberté nécessaire pour mener à terme leurs interventions. "Je possède tout le pouvoir du monde!» (Participant 2, notre traduction de l'anglais). 
Certains participants sont à ce point satisfaits qu'ils expriment une certaine méfiance quant à l'idée d'augmenter leur pouvoir discrétionnaire et de laisser davantage de place à l'évaluation clinique. Un plus grand recours à l'ajustement clinique est dès lors dénoncé du fait qu'il implique un manque de rigueur sur le plan professionnel. Ces participants craignent alors les abus et les erreurs inhérentes à une trop grande liberté d'action dans la pratique évaluative:

Moi, je suis confortable avec le niveau de pouvoir discrétionnaire que j'ai... Je suis satisfaite de la façon que ça fonctionne actuellement... Je pense qu'avoir plus de pouvoir discrétionnaire, il y a possibilité d'abus ou qu'on se trompe. Moi, je considère que j'ai assez de pouvoir discrétionnaire. (Participante 11)

Certaines recherches suggèrent qu'il y a parfois des personnes qui font des évaluations cliniques, et qu'elles les camouflent en évaluations actuarielles... je crois que ce type de pratique est très dangereux. (Participant 2, notre traduction de l'anglais)

Pour d'autres intervenants, le recours aux outils actuariels ne restreint pas leur marge de manœuvre professionnelle, puisque ces outils leur permettent au contraire de faire usage de leur jugement clinique. La satisfaction générale des participants quant au niveau de liberté que leur apporte le recours à des instruments actuariels est d'autant plus grande que plusieurs d'entre eux considèrent qu'il est toujours possible, dans le cadre de leur pratique, d'ajuster les résultats des grilles actuarielles en s'appuyant sur leur propre jugement clinique. «[L'évaluation actuarielle] laisse quand même une place au jugement clinique... au gros bon sens... à l'interaction avec le délinquant» (Participant 11). On enregistre donc chez plusieurs participants une moins grande méfiance à l'égard du recours à l'ajustement clinique:

Je dirais que les résultats actuariels sont importants, mais que j'utilise aussi d'autres informations qui sont à ma disposition... ainsi que le contact personnel que j'établis avec le gars. (Participant 1, notre traduction de l'anglais)

Je serais incapable de faire une évaluation actuarielle sans avoir recours au jugement clinique. Dans certains cas, je sais que les résultats de l'évaluation actuarielle indiqueront un certain niveau de risque, mais mon jugement clinique va m'inciter à ne pas tenir compte de ces résultats. On dira, vous savez, on voit les résultats, mais on juge qu'il y a d'autres facteurs en jeu qui nous inciteront à ajuster le résultat. (Participant 3, notre traduction de l'anglais) 
Le recours à l'ajustement clinique, bien qu'il soit accessible à tous les intervenants qui travaillent avec des grilles actuarielles, est toutefois perçu par certains comme étant un privilège réservé aux intervenants plus expérimentés qui ont eu la possibilité de mieux développer leur expertise clinique:

Je me sens très confiant, très flexible et bien informé dans ce domaine... Mais comme j'ai suivi beaucoup de formations, je reconnais aussi que ce n'est pas tout le monde qui a les ressources que j'ai, et que ces gens sont probablement plus confortables s'ils peuvent justifier leurs décisions à partir des résultats d'une évaluation du risque... Je dirais donc que pour des thérapeutes expérimentés, bénéficier d'un pouvoir discrétionnaire est très important... C'est pourquoi ils sont habiles à répondre à ce qu'ils voient plutôt que de se fier exclusivement aux outils actuariels. (Participant 6 , notre traduction de l'anglais)

La façon dont les participants décrivent le recours à l'ajustement clinique vient donc infirmer notre hypothèse selon laquelle il s'agirait d'une technique de résistance pour contrer le déficit discrétionnaire inhérent à l'évaluation actuarielle. Les intervenants rencontrés considèrent tous que le recours aux grilles actuarielles est un progrès significatif dans le cadre de leur pratique, et que ce progrès est d'autant plus important qu'il n'empêche pas les intervenants d'avoir recours à l'occasion à leur propre jugement clinique. Les intervenants ne considèrent donc pas que le virage actuariel aurait miné de façon importante leur marge de manœuvre professionnelle, en les enfermant dans une fonction purement technique de compilation de données statistiques. Au contraire, le recours à des outils actuariels semble rassurer les intervenants dans leur pratique, tout en leur permettant à l'occasion de mettre à profit leur propre jugement clinique.

\section{Conclusion}

Cette recherche s'appuyait au départ sur l'hypothèse selon laquelle les intervenants appelés à travailler avec des grilles actuarielles manifesteraient une certaine forme de résistance liée au fait que ces outils offriraient très peu de liberté en ce qui concerne le recours au jugement clinique traditionnel. Les résultats de cette étude préliminaire auprès d'intervenants correctionnels semblent infirmer notre hypothèse de départ. En effet, les professionnels rencontrés s'accordent en général pour dire que l'utilisation des outils actuariels ne réduit en rien leur 
pouvoir discrétionnaire, d'autant plus que l'utilisation de ces outils permet toujours le recours à un jugement clinique afin d'ajuster les résultats de ces évaluations. Le recours à des outils actuariels n'est donc pas considéré par les participants comme un obstacle à leur liberté professionnelle. Si on se fie aux propos des intervenants, le recours à l'ajustement clinique ne serait pas utilisé dans une optique de résistance face au virage actuariel, mais plutôt dans une optique de diversification des sources d'information pouvant être utilisées dans le cadre du système de prédiction. L'ajustement clinique n'est pas considéré comme une stratégie permettant de renverser la préséance actuarielle, mais plutôt comme une façon de soutenir et d'améliorer la précision statistique des outils actuariels.

Les résultats de cette étude préliminaire nous permettent aussi de conclure que les outils actuariels semblent bien intégrés dans la pratique correctionnelle canadienne, du moins auprès des intervenants qui travaillent auprès des délinquants sexuels. Les intervenants attribuent à ces nouveaux instruments beaucoup plus d'avantages que d'inconvénients. Ces résultats nous permettent alors d'expliquer (en partie) pourquoi le virage actuariel s'est opéré de façon relativement rapide au sein des institutions correctionnelles canadiennes, ne rencontrant en fait que peu de résistance du côté des principaux acteurs appelés à travailler avec ces nouveaux outils d'évaluation. Il reste à évaluer si le recours à ces outils actuariels, au-delà de son impact sur le caractère technique de l'évaluation, peut contribuer à modifier de façon plus significative les fonctions et les modalités mêmes de la pratique correctionnelle au Canada.

\section{Références}

Andrews, D. A., \& Bonta, J. (1995). The Level of Service Inventory - Revised. Toronto: Multi-Health Systems.

Andrews, D. A., \& Bonta, J. (2003). The Psychology of Criminal Conduct (3th Edition). Cincinnati $(\mathrm{OH})$ : Anderson Publishing Co.

Andrews, D. A., \& Bonta, J. (2006). The Psychology of Criminal Conduct $\left(4^{\text {th }}\right.$ Edition). Cincinnati $(\mathrm{OH})$ : Anderson Publishing Co.

Andrews, D. A., Bonta, J., \& Wormith, J. S. (2006). The Recent Past and Near Future of Risk and/or Need Assessment. Crime and Delinquency, 52 (1), 7-27.

Beech, A. R., Fisher, D. D., \& Thornton, D. (2003). Risk Assessment of Sex Offenders. Professional Psychology: Research and Practice, 34 (4), 339-352.

Bonta, J. (1996). Risk-Needs Assessment and Treatment. In A. T. Harland (Ed.), Choosing Correctional Options That Work (18-32). Thousand Oaks (CA): Sage. 
Briggs, P. F., \& Wirt, R. D. (1965). Prediction. In H. C. Quay (Ed.), Juvenile Delinquency: Research and Theory (170-208). Princeton (NJ): Van Nostrand.

Cartuyvels, Y. (2008). Les ambivalences du risque: Regards croisés en sciences sociales. Bruxelles: Facultés universitaires Saint-Louis.

Cheliotos, L. K. (2006). How Iron is the Iron Cage of New Penology? The Role of Human Agency in the Implementation of Criminal Justice Policy. Punishment and Society, 8 (3), 313-340.

Dawes, R. M., Faust, D., \& Meehl, P. E. (1989). Clinical Versus Actuarial Judgment. Science, 243, 1668-1674.

Feeley, M., \& Simon, J. (1994). Actuarial Justice: The Emerging New Criminal Law. In D. Nelken (Ed.), The Futures of Criminology (173-201). London: Sage.

Feeley, M., \& Simon, J. (1992). The New Penology: Notes on the Emerging Strategy of Corrections and its Implications. Criminology, 30 (4), 449-474.

Fitzgibbon, D. W. M. (2007). Risk Analysis and the New Practitioner. Myth or Reality? Punishment and Society, 9 (1), 87-97.

Glaser, D. (1985). Who Gets Probation and Parole: Case Study Versus Actuarial Decision Making. Crime and Delinquency, 31 (3), 367-378.

Gottfredson, M. R. \& Gottfredson, D. M. (1988). Decision Making in Criminal Justice: Toward the Rational Exercice of Discretion. New York: Plenum Press.

Grove, W. M., \& Meehl, P. E. (1996). Comparative Efficiency of Informal (Subjective, Impressionnistic) and Formal (Mechanical, Algorithmic) Prediction Procedures: The Clinical-Statistical Controversy. Psychology, Public Policy, and Law, 2 (2), 293-323.

Grove, W. M., Zald, D. H., Lebow, B. S., Snitz, B. E., \& Nelson, C. (2000). Clinical Versus Mechanical Prediction: A Meta-Analysis. Psychological Assessment, 12 (1), 19-30.

Grubin, D. (2004). The Risk Assessment of Sex Offenders. In H. Kemshall \& G. McIvor (Eds.), Managing Sex Offender Risk (91-110). London: Jessica Kingsley Publishing.

Guay, J. P. (2006). Prédiction actuarielle et prédiction clinique: le dernier souffle d'une pratique traditionnelle. Revue internationale de criminologie et de police technique et scientifique, 59, 149-164.

Hacking, I. (1990). The Taming of Chance. Cambridge (UK): Cambridge University Press.

Hannah-Moffat, K. (2005). Criminogenic Needs and the Transformative Risk Subject: Hybridizations of Risk/Need in Penalty. Punishment and Society, 7 (1), 29-51.

Hare, R. D. (1996). Psychopathy: A Clinical Construct Whose Time Has Come. Criminal Justice and Bebavior, 23 (1), 25-54.

Harris, G. T., Rice, M. E., \& Quinsey, V. L. (1993). Violent Recidivism of Mentally Disorded Offenders: The Development of a Statistical Prediction Instrument. Criminal Justice and Behavior, 20 (4), 315-335.

Harris, P. M. (2006). What Community Supervision Officers Need to Know About Actuarial Risk Assessment and Clinical Judgment. Federal Probation, $70(2), 8-14$. 
Hart, S. D., Michie, C., \& Cooke, D. J. (2007). Precision of Actuarial Risk Assessment Instruments. Evaluating the Margin of Error of Group v. Individual Prediction of Violence. British Journal of Psychiatry, 190 (49), 60-65.

Hemphill, J. F. \& Hare, R. D. (2004). Some Misconceptions About the Hare PCL-R and Risk Assessment. Criminal Justice and Bebavior, 31 (2), 203-243.

Horlick-Jones, T. (2005). On Risk Work: Professional Discourse, Accountability, and Everyday Action. Health, Risk and Society, 7 (3), 293-307.

Kemshall, H. (2003). Understanding Risk in Criminal Justice. Maidenhead (UK): Open University Press.

Litwack, T. R. (2001). Actuarial Versus Clinical Assessments of Dangerousness. Psychology, Public Policy, and Law, 7 (2), 409-443.

Litwack, T. R., \& Schlesinger, L. B. (1999). Dangerousness Risk Assessments: Research, Legal, and Clinical Considerations. In A. K. Hess \& I. B. Weiner (Eds.), The Handbook of Forensic Psychology (171-217). New York: Wiley.

Meehl, P. (1954). Clinical vs Statistical Prediction: A Theoretical Analysis and a Review of the Evidence. Minneapolis: University of Minnesota Press.

Maurutto, P., \& Hannah-Moffat, K. (2006). Assembling Risk and the Restructuring of Penal Control. British Journal of Criminology, 46, 438-454.

Monahan, J. (1981). Predicting Violent Behavior: An Assessment of Clinical Techniques. Beverly Hills (CA): Sage.

Pham, T. H., \& Côté, G. (2000). Psychopathie: théorie et recherche. Paris: Presses universitaires du Septentrion.

Pratt, J. (1997). Governing the Dangerous: Dangerousness, Law, and Social Change. Sydney (Australia): The Federation Press.

Quinsey, V. L., Harris, G. T., Rice, M. E., \& Cormier, C. A. (2006). Violent Offenders: Appraising and Managing Risk (2 ${ }^{\text {nd }}$ Edition). Washington (DC): American Psychological Association.

Quirion, B. (2006). Traiter les délinquants ou contrôler les conduites: le dispositif thérapeutique à l'ère de la nouvelle pénologie. Criminologie, 39 (2), 137-164.

Quirion, B. (2009). Le détenu autonome et responsable: la nouvelle cible de l'intervention correctionnelle au Canada. Revue de droit pénal et de criminologie, juillet-août 2009, 818-835.

Rose, N. (2002). At Risk of Madness. In T. Baker \& J. Simon (Eds.), Embracing Risk: The Changing Culture of Insurance and Responsibility (209-237). Chicago: University of Chicago Press.

Vacheret, M., Dozois, J., \& Lemire, G. (1998). Le système correctionnel canadien et la nouvelle pénologie: la notion de risque. Déviance et société, 22 (1), 37-50.

Walker, S. (1993). Taming the System: The Control of Discretion in Criminal Justice (1950-1990). Oxford: Oxford University Press. 
ABSTRACT - Within the framework of this article, we will be addressing the question of the actuarial shift that occurred in the field of corrections, particularly with regard to the multiplication of actuarial risk assessment tools, which are at the disposal of correctional professionals. The objectives of this article are threefold. Firstly, we describe the extent and the nature of the transformations that took place during the last two decades with regard to the tools used by Canadian correctional professionals. Thereafter, in our conceptual and theoretical framework, we outline the principal issues of these technical transformations, particularly with regard to the reduction of discretionary power granted to these professionals. Finally, we analyze the discourse of these professionals in an effort to further evaluate how the professionals themselves received the impact of those modifications.

KEYWORDS - Correctional intervention, risk assessment, actuarial tools, discretionary power, Canada.

RESUMEN - En este artículo abordamos el viraje actuarial que se manifiesta en el campo penitenciario, en particular la multiplicación de herramientas de evaluación actuarial a disposición de los funcionarios penitenciarios. Los objetivos del artículos son tres. Se describe primero la naturaleza de los cambios operados en el curso de los pasados decenios en materia de herramientas empleadas por los funcionarios de las cárceles canadienses. A continuación se delimitan en el plano conceptual y teórico las principales implicaciones de estas transformaciones técnicas, en particular respecto de la reducción del poder discrecional acordado a los funcionarios penitenciarios. Por último, se analiza el discurso de los funcionarios con el fin de evaluar la forma en que estas transformaciones se perciben en el marco de la práctica profesional de estos cuadros. Se trata de hecho de evaluar el impacto de la introducción y utilización de una nueva gama de herramientas actuariales para las prácticas correccionales.

PALABRAS CLAVE - Intervención correccional, evaluación de riesgo, herramientas actuariales, poder discrecional, Canadá. 